\title{
A case of primary psoas abscess presenting as buttock abscess
}

\author{
Jae Ho Yoo • Eung Ha Kim • Hyun Seok Song • \\ Jang Gyu Cha
}

Received: 29 January 2009/Accepted: 23 October 2009/Published online: 20 November 2009

(C) Springer-Verlag 2009

\begin{abstract}
Buttock abscess is a rare clinical manifestation from unusual extrapelvic extension of psoas abscess. A 48-year-old woman presented with painful swelling of the buttock with a sense of local heat. Magnetic resonance imaging revealed a large subfascial abscess over the glutei muscles and was traced into the intraabdominal cavity over the iliac wing to the psoas muscle. Both the psoas abscess and the buttock abscess were evacuated via separate approaches. Empirical antibiotic therapy was delivered for 3 weeks. After 6 months, no evidence of recurrence was found. Psoas abscess could be included in the differential diagnosis of buttock abscess.
\end{abstract}

Keywords Psoas abscess · Buttock abscess

\section{Introduction}

Abscess of the psoas muscle is rare [1, 2]. Clinical presentation includes back pain with limited function, fever, and leukocytosis [3]. Prompt diagnosis continues to rely upon retaining a high degree of suspicion, as the signs and symptoms may be diffuse, chronic, and nonspecific [4-7]. Because the psoas musculature extends from the lowest thoracic and five lumbar vertebrae to the lesser trochanter of the femur, differentiation from the pathologies around

J. H. Yoo $(\bowtie) \cdot$ E. H. Kim · H. S. Song

Department of Orthopaedic Surgery,

Soonchunhyang University Hospital, 1174 Jung-dong,

Wonmi-gu, Bucheon, Gyeonggi-do 420-767, South Korea

e-mail: yoojaeho@hotmail.com; jadeboykr@gmail.com

J. G. Cha

Department of Radiology, Soonchunhyang University Hospital,

Bucheon, Gyeonggi-do, South Korea the hip joint is emphasized [8-14]. The muscle itself sometimes acts as a conduit for the spread of suppuration, and propagations to the hip joint [8, 15-17], the thigh [18], and even the calf [4] have been reported. However, only a few cases of unusual spread of psoas abscess to the flank $[3,19]$ or buttock [20] have been described. It would not be so easy for a clinician to suspect the presence of the psoas abscess if the initial manifestation were a flank or buttock mass.

We present a case of psoas abscess, the initial clinical presentation of which was painful swelling of the buttock with local erythema and a sense of heat. Recognition of the psoas abscess as well as the apparent manifestation and evacuation of the whole lesion via combined anterior retroperitoneal approach and direct incision over the buttock led to complete eradication of the pathology.

\section{Case report}

A 48-year-old woman presented with painful swelling of the buttock with a sense of localized heat. It had developed 1 month earlier and aggravated 1 week prior to presentation. The lesion was located in the upper lateral quadrant of the left buttock and was $10 \mathrm{~cm}$ in diameter, with erythema and localized heat. There were only mild generalized symptoms, with elevation of body temperature to $38.1^{\circ} \mathrm{C}$. She had no notable past medical or medication history, such as diabetes, hypertension, or tuberculosis, and had not undergone any surgery. She had no local injection in the buttock for several months prior to presentation. Blood tests showed a borderline increased white blood cell count $9,600 / \mathrm{mm}^{3}$ and elevated C-reactive protein level of $10.14 \mathrm{mg} / \mathrm{dl}$. Cellulitis with or without abscess formation of the buttock was suspected, and magnetic resonance 

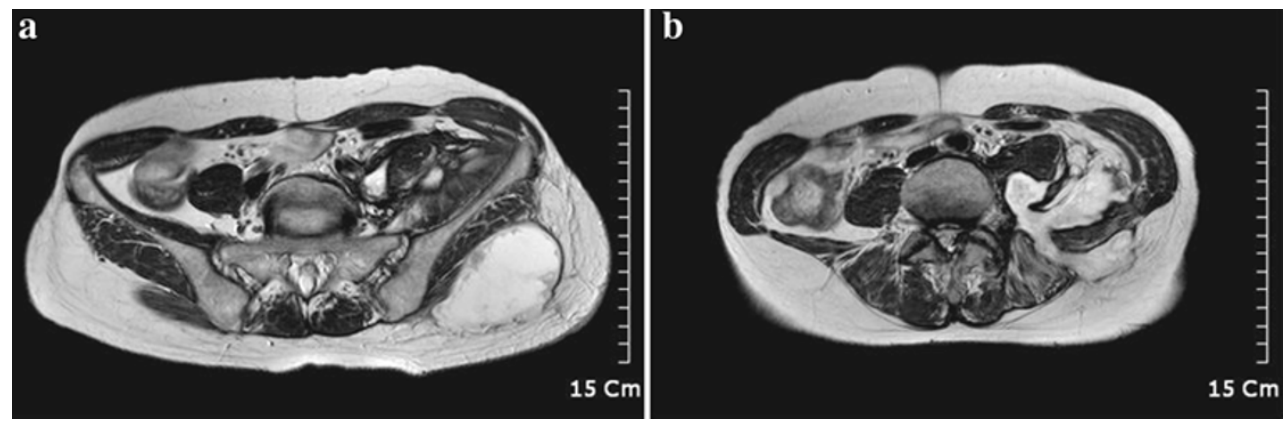

Fig. 1 Axial magnetic resonance images show the extent of the abscess pocket. The manifest painful swelling of the buttock is due to subfascial pus collection (a), and the purulent fluid is connected to the intra-abdominal retroperitoneal and psoas abscess (b)

(MR) images were examined to determine the extent of the pathology. MR imaging revealed a large subfascial abscess over the glutei muscles, and the abscess was traced to the intra-abdominal cavity over the iliac wing. The communication of the buttock abscess into the retroperitoneum and the psoas abscess was evident. The defect of the abdominal wall was through the sacrospinalis, or erector spinae and the internal oblique abdominal musculature (Fig. 1). Pathologies of adjacent organs such as vertebrae, kidney, ureter, and bowel were excluded.

Operative evacuation was planned on both the buttock and psoas abscess. For the buttock abscess, direct skin incision over the lesion was sufficient to eliminate the pathology, whereas the anterolateral retroperitoneal approach was applied to eradicate the retroperitoneal and psoas abscess (Fig. 2). Several specimens were taken to identify the causative organism. However, results of the ordinary bacterial examination as well as anaerobes, fungi, and tuberculosis were negative. Cytology for malignant cells was also negative, and histology examination revealed no caseation necrosis implying tuberculosis. Empirical antibiotic therapy with first-generation cephalosporin was initiated and administered for 3 weeks. Without subjective symptom and the decreasing trend of the hematologic inflammation marker such as C-reactive protein reaching normal range, the antibiotics were discontinued. After 6 months of being antibiotic free, the patient had no evidence of recurrence upon an outpatient department visit. She returned to activities of daily living without difficulty. An informed consent to publish her case was provided.

\section{Discussion}

The psoas abscess is rare [1, 2], and those presenting as extrapelvic extension are even more rare. The psoas abscess that extends over the iliac wing and presents as a flank or buttock abscess is a much rarer clinical feature, with only a few case reports in the medical literature $[3,9$,

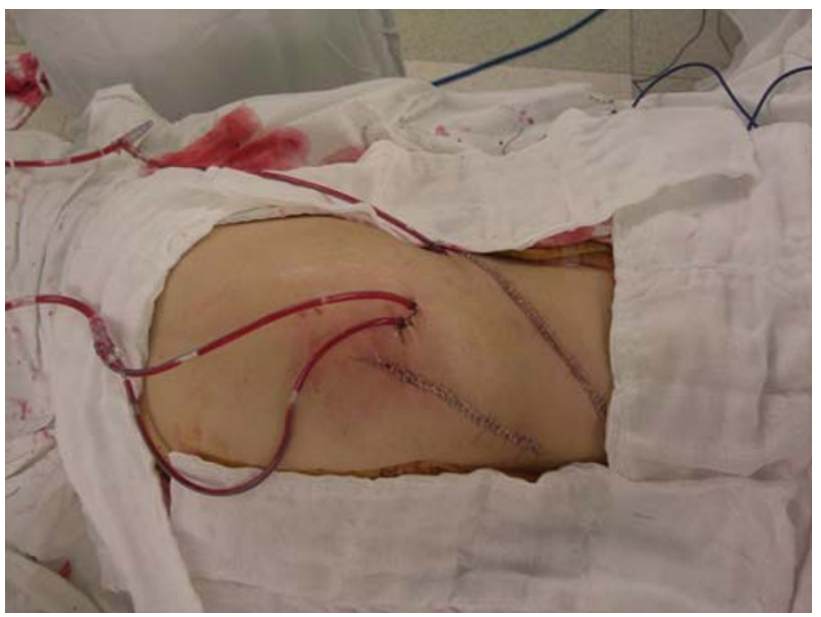

Fig. 2 The apparent buttock abscess was eliminated by direct approach over the lesion below the iliac wing, and the psoas and retroperitoneal abscess was eradicated by anterolateral retroperitoneal approach above the iliac wing. The closed suction drain was inserted into the retroperitoneum through the communicating channel between the buttock and the psoas lesions

19-22]. Although the worldwide incidence was considered to be only 12 cases per year [1,2], it is more frequently diagnosed and reported with the advent of computed tomography (CT) and MR imaging [2, 23]. The psoas abscess is considered primary if the cause is hematogenous seeding from a distant site and secondary if there is a contiguous infectious source from vertebrae, pancreas, kidney, ureter, appendix, bowel, or hip joint $[1,2,24]$. Our case was primary psoas abscess, which extends to the adjacent retroperitoneum and buttock through the abdominal wall. The susceptibility of the psoas muscle as primary type is attributed to its rich blood supply and proximity to overlying retroperitoneal lymphatic channels $[25,26]$.

Because the iliopsoas muscle inserts onto the lesser tubercle of the proximal femur, it is possible that the psoas abscess could extend to the medial side of the thigh under the inguinal canal [18] or to the hip joint [8, 15-17]. Extension onto the calf has also been reported [4]. However, 
extension of the psoas abscess to the buttock over the iliac wing is an extremely rare clinical manifestation. Local spread into the retroperitoneum from the psoas abscess and to the extrapelvic extension over the iliac wing through the weak abdominal wall lateral to the paraspinal musculature could be speculated in our case. Moreover, piriformis and gluteal abscesses have also been reported [21] (Fig. 3). The clinical relevance of our case is that the psoas abscess could be a rare cause of buttock abscess. It deserves the caution of the clinician all-the-more in that the detection of the hidden psoas abscess requires high level of suspicion and that failure to recognize and treat psoas abscess results in considerable morbidity [27].

The most common causative organism of the psoas abscess is Staphylococcus aureus in primary type and enteric organisms in secondary type [2, 4, 24], whereas tuberculous spinal infection was the most common etiology half a century ago [27]. Fungal infection also should be excluded $[4,23,28]$. In addition to the origin of infection, appendicitis, pancreatitis, pyelonephritis, and Crohn's disease occasionally cause psoas abscess [25, 27]. The pathogenic organism could not be identified in our case. Because the negative result of the microbiological test cannot warrant the absence of pathogen, broad-spectrum antibiotics were empirically delivered.

This is not the first case in the medical literature in which the psoas abscess presented as a flank or buttock

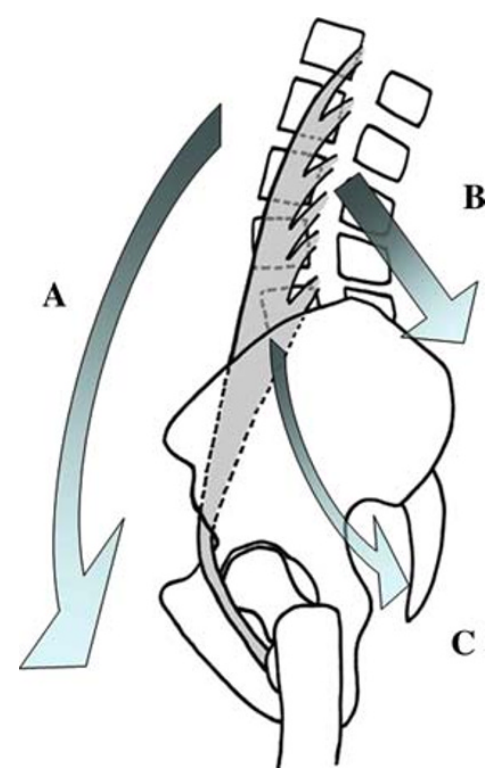

Fig. 3 Spread of psoas abscess. Usual propagation of the abscess in the psoas along the insertion of the muscle into the lesser trochanter of the femur, then the medial side of the thigh, and even downward to the calf $(A)$. Unique blowout of the psoas abscess in this case. The abscess expanded into the retroperitoneum, outside of the psoas muscle substance, and then reached the buttock through the posterior abdominal wall over the iliac crest $(B)$. Psoas abscess may result in piriformis and gluteal abscess $(C)$ mass. Mycobacterial [3, 19] or pyogenic [9] infection of the psoas muscle has been reported to propagate to the retroperitoneum or flank/buttock through the abdominal wall. Psoas abscess and cellulitis of the right gluteal region resulting from retroperitoneal perforation of cecum carcinoma has also been reported [22]. Psoas, piriformis, and gluteal abscess, as well as right iliac vein thrombosis, have been documented [21].

We document a rare clinical manifestation of buttock abscess from unusual extrapelvic extension of a psoas abscess. Psoas abscess could be included in the differential diagnosis of buttock abscess.

Conflict of interest statement The authors declare that they have no conflict of interest related to the publication of this manuscript.

\section{References}

1. Gruenwald I, Abrahamson J, Cohen O (1992) Psoas abscess: case report and review of the literature. J Urol 147(6):1624-1626

2. Ricci MA, Rose FB, Meyer KK (1986) Pyogenic psoas abscess: worldwide variations in etiology. World J Surg 10(5):834-843

3. Rawdha T, Leila S, Hadj Yahia Chiraz B, Leila A, Lilia C, Rafik $\mathrm{Z}$ (2008) A rare cause of flank mass: tuberculous psoas abscess. Intern Med 47(10):985-986

4. Garner JP, Meiring PD, Ravi K, Gupta R (2007) Psoas abscessnot as rare as we think? Colorectal Dis 9(3):269-274

5. Maradiaga GM, Fiorilli MG, Elmore WG, Hunt J (1998) Psoas abscess. Diagnosed if suspected. N C Med J 59(1):54-56

6. Toren A, Ganel A, Lotan D, Graif M (1989) Delayed diagnosis of a primary psoas abscess mimicking septic arthritis of the hip. J Pediatr Surg 24(2):227-228

7. Yin HP, Tsai YA, Liao SF, Lin PH, Chuang TY (2004) The challenge of diagnosing psoas abscess. J Chin Med Assoc 67(3):156-159

8. Buttaro M, Gonzalez Della Valle A, Piccaluga F (2002) Psoas abscess associated with infected total hip arthroplasty. J Arthroplasty $17(2): 230-234$

9. Ebraheim NA, Rabenold JD, Patil V, Sanford CG (2008) Psoas abscess: a diagnostic dilemma. Am J Orthop 37(1):E11-E13

10. Hamilton J, Wilson H, Capell H, McInnes I, Madhok R (2001) The hip or not. J Rheumatol 28(6):1398-1400

11. Perry J, Barrack RL, Burke SW, Haddad RJ Jr (1985) Psoas abscess mimicking a septic hip. Diagnosis by computed tomography. J Bone Joint Surg Am 67(8):1281-1283

12. Simons GW, Sty JR, Starshak RJ (1983) Retroperitoneal and retrofascial abscesses. A review. J Bone Joint Surg Am 65(8):1041-1058

13. Song J, Letts M, Monson R (2001) Differentiation of psoas muscle abscess from septic arthritis of the hip in children. Clin Orthop Relat Res 39(1):258-265

14. Stefanich RJ, Moskowitz A (1987) Hip flexion deformity secondary to acute pyogenic psoas abscess. Orthop Rev 16(2):6777

15. Compain C, Michou L, Orcel P, Hannouche D, Richette P (2008) Septic arthritis of the hip with psoas abscess caused by non-typhi Salmonella infection in an immunocompetent patient. Joint Bone Spine 75(1):67-69

16. Kumagai K, Ushiyama T, Kawasaki T, Matsusue Y (2005) Extension of lumbar spine infection into osteoarthritic hip through psoas abscess. J Orthop Sci 10(1):91-94 
17. Liu S, Leung H, Kadow C, Obrien JM (1992) Septic arthritis of the hip complicating Salmonella psoas abscess. Case report. Scand J Urol Nephrol 26(3):305-306

18. Prassopoulos PK, Giannakopoulou CA, Apostolaki EG, Charoulakis NZ, Gourtsoyiannis NC (1998) Primary ilio-psoas abscess extending to the thigh in a neonate: US, CT and MR findings. Pediatr Radiol 28(8):605-607

19. Sanal HT, Kocaoglu M, Sehirlioglu A, Bulakbasi N (2006) A rare cause of flank mass: psoas abscess due to extensive primary thoracolumbar tuberculous spondylodiskitis. AJNR Am J Neuroradiol 27(8): 1735-1737

20. Spedding RL, Walsh IK (1999) Retroperitoneal abscess presenting with a buttock swelling and anaemia. J Accid Emerg Med 16(4):302-303

21. Arai Y, Kawakami T, Soga H, Okada Y (1999) Psoas abscess associated with iliac vein thrombosis and piriformis and gluteal abscesses. Int J Urol 6(5):257-259

22. Kobayashi H, Sakurai Y, Shoji M, Nakamura Y, Suganuma M, Imazu H, Hasegawa S, Matsubara T, Ochiai M, Funabiki T (2001)
Psoas abscess and cellulitis of the right gluteal region resulting from carcinoma of the cecum. J Gastroenterol 36(9):623-628

23. Yacoub WN, Sohn HJ, Chan S, Petrosyan M, Vermaire HM, Kelso RL, Towfigh S, Mason RJ (2008) Psoas abscess rarely requires surgical intervention. Am J Surg 196(2):223-227

24. Taiwo B (2001) Psoas abscess: a primer for the internist. South Med J 94(1):2-5

25. Ramus NI, Shorey BA (1975) Crohn's disease and psoas abscess. Br Med J 3(5983):574-575

26. Santaella RO, Fishman EK, Lipsett PA (1995) Primary vs. secondary iliopsoas abscess. Presentation, microbiology, and treatment. Arch Surg 130(12):1309-1313

27. Procaccino JA, Lavery IC, Fazio VW, Oakley JR (1991) Psoas abscess: difficulties encountered. Dis Colon Rectum 34(9):784 789

28. Perros P, Sim DW, MacIntyre D (1988) Psoas abscess due to retroperitoneal tuberculous lymphadenopathy. Tubercle 69(4): 299-301 